\title{
Féminisation de l'épidémie de VIH/sida et actions de terrain
}

\author{
Danièle AUTHIER ${ }^{1}$, Carine FAVIER ${ }^{2}$
}

\begin{abstract}
Résumé
Devant la féminisation de l'épidémie de VIH et l'absence d'une prévention spécifique en direction des femmes, deux actions sont mises à place pour expérimenter une nouvelle approche préventive qui intègre la question du genre et de la réduction des risques. Plaçant les personnes au centre de la démarche, les programmes privilégient l'ouverture d'espaces de paroles et d'échanges de savoir pour renforcer les compétences des femmes et réduire leur exposition à l'ensemble des risques liés à la sexualité.
\end{abstract}

Mots clés : réduction des risques, genre, sexualité, rapports sociaux de sexe, santé sexuelle.

\section{Femmes et VIH : le contexte des années 1990}

\section{Une épidémie qui se féminise}

Dès le début des années 1990, les données épidémiologiques soulignent l'augmentation du nombre de femmes contaminées dans le monde : le $1^{\text {er }}$ décembre 1990 , l'OMS consacre sa troisième journée mondiale au thème "les femmes et le sida ". Au même moment, en France, "pour répondre aux préoccupations exprimées par les femmes à partir de leurs propres questions", l'Agence française de lutte contre le sida et le Centre national d'information sur les droits des femmes et des familles " unissent leurs compétences " pour publier une brochure "Prévention du sida : questions de femmes ". Celle-ci fait état de $17 \%$ de femmes parmi les personnes atteintes du sida en France [1]. Dans les années qui suivent, entre 1991 et 1996, le sex ratio est de 4,4 parmi les cas de sida diagnostiqués [2]. Face à ce constat, en 1996, la division sida de la Direction générale de la santé, sollicite le service des droits des femmes, le Conseil supérieur de l'information sexuelle (CSIS), la Coordination des associations pour le droit à l'avortement et à la contraception, le Mouvement français pour le planning familial et les associations de lutte contre le sida pour envisager les conditions d'une stratégie nationale de prévention de l'infection à VIH en direction des femmes. Cette étape de concertation aboutit, en novembre 1997, au colloque "Femmes et infection à VIH en Europe ", à Paris ${ }^{3}$. Dépassant le champ de la transmission mère-enfant, l'influence des rapports sociaux de sexe ou de genre sur les difficultés de prévention des femmes est mise en avant dans les recommandations. Le suivi de la cohorte européenne de femmes séropositives [3] met en évidence le lien entre transmission hétérosexuelle de l'épidémie, conditions sociales et rôle attendu des femmes ou, autrement dit, du sens qu'elles donnent à la sexualité dans le monde où elles vivent. Ainsi, $75 \%$ des cas féminins de sida en Europe sont enregistrés en France, en Espagne, en Italie, dans des pays où les droits des femmes ont connu des avancées significatives, sans que cela implique, au vu des données épidémiologiques, une mise à distance des codes de conduite sexuelle et amoureuse, qui représentent une "force motrice de l'épidémie " [4]. On retrouve ces mêmes données dans les départements français d'Amérique [5] et, plus largement, sur le plan international, dans les pays du Sud.

Parallèlement, au niveau international, l'efficacité des actions et campagnes qui s'inscrivent dans une démarche globale de " santé sexuelle " associant sida et reproduction semble démontrée [6-8]. Cette évolution apparaît également dans l'organigramme de la division sida du ministère de la Santé en 1996, l'intitulé " Femmes et sida ", remplaçant " Mères/Enfants/Sida ", faisant apparaître la cible " femmes » comme autonome et substituant au concept de santé reproductive au centre des décisions et programmes sur la santé des femmes, à celui, plus large et plus opportun, à tous égards, de " santé sexuelle " [9].

La question est alors posée de la nécessité, en complément d'une approche généraliste et grand public, d'une stratégie spécifique en direction des femmes. Très concrètement, il s'agit de mettre en place des actions en direction des femmes qui intègrent l'asymétrie des rapports sociaux de sexe ou de genre, les violences et les inégalités en tant que facteurs d'entrave à l'information et à la protection des rapports sexuels.

\footnotetext{
Programme a Femmes, réduction des risques et sexualité * (Frisse), Lyon. 2 Programme Réduction des risques sexuels, Mouvement français pour le planning
} familial.
Organisé, par la division sida de la Direction générale de la santé, en partenariat avec
Sida info service et le Centre européen de surveillance épidémiologique du sida. 


\section{Des stratégies de prévention indifférenciées}

Alors que les enjeux de la prévention au féminin viennent questionner les représentations des femmes qui prennent l'initiative de la protection de la relation sexuelle, les acteurs de terrain ne disposent que d'un seul « outil technique ", le préservatif masculin, présenté comme une méthode de protection facile à gérer par toutes et tous. Or, en France, les préservatifs masculins ne sont réintroduits dans la publicité qu'en janvier 1987, c'est-à-dire en pleine " homo sexualisation » du sida [10], et 20 ans seulement après l'arrivée de la pilule et la médicalisation de la contraception, qui renvoie aux oubliettes l'efficacité contraceptive du préservatif masculin ${ }^{4}$ [11]. Remettant en cause la « liberté sexuelle " obtenue avec la maîtrise de la contraception par les femmes, la prévention du sida les oblige à envisager leur partenaire comme un danger potentiel, brisant le rêve du modèle amoureux $[12,13]$. Comment les femmes peuvent-elles alors ne pas se sentir désarmées, voire culpabilisées, de ne pas arriver à "négocier " ou " imposer » des rapports protégés?

Quant au préservatif féminin ${ }^{5}$, son agrément est le sujet de nombreux débats, de 1991 à 1997, entre l'Association française de normalisation (Afnor) et I'Agence française de lutte contre le sida, jusqu'à son homologation le 18 juin 1998. Sa récente mise à disposition peut expliquer, pour partie, les résistances des femmes à son égard : tout aussi visible que son homologue masculin, son port n'est pas neutre puisqu'il met en scène, en quelque sorte, le droit des femmes à prendre l'initiative et la responsabilité de la protection, tout en courant le risque de se montrer " disponible ". Le rapport des femmes aux techniques de prévention existantes ne peut donc se faire sans aborder la question des modèles masculin/féminin et des rapports de domination hommes/femmes, si l'on veut permettre aux femmes de renforcer leurs capacités à faire acte de prévention

\section{Introduire la notion de genre pour renforcer les compétences des femmes : deux actions pour mettre en œuvre cet objectif}

C'est dans ce contexte que le Mouvement français pour le planning familial et l'association « Femmes, réduction des rlsques et sexualité " (Frisse), ont choisi de développer des actions spécifiques qui prennent en compte la question des rapports sociaux de sexe ou de genre.

Deux programmes ont été développés à partir de bases communes, historique et politique : s'appuyant sur l'expérience de l'action menée par l'Équipe mobile d'information et de pré-

\footnotetext{
Rappelons que jusqu'à l'arrivée de la pilule, les hommes prenaient part au contrôle des naissances au moyen du retrait ou du préservatif.

Disponible en Europe à partir de 1992 (Autriche, Grèce, Pays Bas, Espagne, Suisse Grande-Bretagne) et à partir de 1993 aux Etats-Unis (agrément par la Food and Drug Administration).
}

vention du sida des Bouches-du-Rhône autour du programme "Femmes, mon corps et moi ». Les deux programmes formulent deux hypothèses qu'ils vont mettre à l'épreuve des faits. Premièrement, s'appuyer sur une démarche de réduction des risques permet d'augmenter les marges de manœuvre des femmes. II s'agit avant tout de postuler que l'information est un acte de prévention et de parler autant des difficultés rencontrées avec les préservatifs masculins et féminins qu'avec d'autres méthodes barrières (comme le diaphragme) ayant démontré leur potentiel à réduire le risque de maladies sexuellement transmissibles et de grossesses non désirées. Invisibles pour le partenaire, elles sont utiles pour toutes les femmes qui, dans des situations et à des moments différents de leur vie, ne peuvent ou ne veulent pas imposer un préservatif, sans pour autant les contraindre à renoncer à une relation sexuelle qu'elles désirent.

La sexualité relevant de la sphère privée, le parti pris est de travailler, dans un premier temps, uniquement avec des femmes, pour faciliter la parole sur les représentations du plaisir et du désir au féminin et leurs implications dans la "culture de prévention du sida " [14].

Évoquée généralement en terme de "vulnérabilité ", la situation des femmes face au VIH est présentée, dans les deux programmes, en termes d'exposition aux risques, partant de l'idée que les femmes ont toutes un potentiel d'action. C'est ce potentiel que les deux programmes cherchent à développer avec et auprès des femmes.

\section{Le programme Frisse}

Ce programme est né dans la dynamique du colloque de 1997 , de la mobilisation de lyonnaises pour la plupart impliquées dans les luttes pour les droits des femmes. Le cadre et le contenu du programme résultent de la mutualisation de leurs apports théoriques et pratiques : médecine, sociologie, psychologie sociale, sexologie, expériences professionnelles et/ou militantes dans la lutte contre le sida. Au départ, l'objectif est de faire connaître aux professionnels les facteurs physiologiques et sociaux qui aggravent les risques de maladies sexuellement transmissibles pour les femmes, afin que ces informations soient relayées auprès de leurs publics. Les deux rencontres organisées dans ce but rassemblent plus de 120 professionnel-le-s, dont une majorité de femmes. A cette occasion, les professionnelles sont nombreuses à exprimer le besoin d'une formation plus approfondie, ouverte uniquement à des femmes, apportant des informations précises sur les risques physiologiques et sociaux, et permettant de découvrir des expériences d'actions avec et auprès des femmes. Cette seconde étape aboutit à la mise en place de « formationsactions " (huit jours répartis sur trois semaines), qui restent, en 2007, l'action pivot de ce programme. Cette appellation souligne une volonté de mobilisation et de transmission des messages auprès de l'entourage familial et/ou professionnel. C'est pourquoi, les participantes peuvent s'inscrire à titre professionnel 
ou personnel. L'idée est de considérer que toutes les femmes ont acquis un savoir profane sur la sexualité et de confronter ces savoirs à des apports théoriques ou professionnels pour que toutes en tirent un bénéfice. Ce choix est un défi sans cesse renouvelé. II aboutit à la constitution de groupes hétérogènes composés de femmes d'origines ethniques, d'âges et de statuts sociaux différents.

Le premier thème abordé est donc ce qu'elles ont en commun : leur sexe anatomique. Le rapport au corps relevant d'une construction sociale, les intervenantes, médecins, abordent toutes les manières d'en prendre soin : toilette intime, examen gynécologique, outils de réduction des risques de maladies sexuellement transmissibles et de grossesses non désirées. De la mise en avant de cette thématique résulte une " panoplie d'outils " qui s'enrichit, au fil des ans, d'objets amenés par des participantes ou des intervenantes, qui sont utilisés comme autant de " facilitateurs " de parole. Ainsi, au-delà de la fonction préventive qui lui est assignée, la "digue dentaire " ou Dim Dam Dom, sert à parler des rapports bouche-sexe entre un homme et une femme, ou entre deux femmes, du plaisir, des représentations du rapport sexuel et des risques de maladies sexuellement transmissibles par d'autres voies que la pénétration vaginale ou anale. Le droit des femmes au plaisir est évoqué avec les vibromasseurs : de leurs ancêtres imaginés pour "soigner » l'hystérie [15] à leurs usages actuels, en passant par la signification de l'excision ou de la clitorectomie. De la même manière, les rapports sociaux de sexe et la construction sociale de l'amour sont abordés à partir d'apports théoriques et retravaillés autour de supports visuels qui sont autant d'occasions de débats.

Deux autres thématiques centrales sont également développées : 1) les risques accrus de rapports non protégés liés à la consommation de substances psycho-actives légales ou illégales ; 2 ) la connaissance de lieux ressources (dépistage, soins, violences sexuelles et conjugales, droits, techniques de défense...) La question de la connaissance du réseau de dépistage et d'accompagnement aux droits et aux soins est abordée sous deux angles: d'une part une journée "table ronde " permettant à chaque participante de présenter ses missions professionnelles et/ou son projet personnel; d'autre part une présentation par des professionnelles de leurs actions (violences sexuelles, prévention jeunes, dépistage). La formation-action constitue une " action pivot " puisque, dès 1999, les participantes décident de poursuivre le travail amorcé : accompagnement de projets, journées thématiques, actions interpartenariales. Le résultat est un pôle multisectoriel de personnes ressources, qui offre un large spectre de territoires d'intervention: lieux et structures diversifiées (associations de quartier, maisons des jeunes et de la culture, clubs de prévention, centres de planification et d'éducation familiale, centres d'hébergement et de réinsertion sociale, groupes militants), mais aussi toute une gamme «d'identités sexuelles $»$.

\section{Le programme Réduction des risques sexuels}

Le programme Réduction des risques sexuels du Mouvement français pour le planning familial est également issu du colloque de 1997. II a commencé fin 1998 et s'est déroulé pendant les deux premières années au sein du Mouvement français pour le planning familial. II a été l'occasion de confirmer, comme le souligne le rapport d'évaluation de Janine Mossuz-Lavau [16], "que les femmes ont très peu d'espaces de parole pour parler d'elles, de leurs questionnements autour de leur vécu du plaisir ou de l'absence de plaisir, de leur désir. Elles ont soif de découvrir un corps qu'elles ne connaissent pas et éprouvent beaucoup de joie à échanger avec les autres femmes au sein d'un groupe, dès que la confiance est instaurée ». Dès 2001, il a été proposé à des femmes, actrices de prévention au sein d'associations ou d'institutions diverses, d'intégrer des formations à l'animation du programme Réduction des risques sexuel. Des associations de lutte contre le sida, des centres d'hébergement et de réinsertion sociale, des associations communautaires, des centres de Protection maternelle et infantile, des associations de prévention auprès des usagers de drogues, des associations de quartier ou autour de l'insertion (alphabétisation, accueil des personnes étrangères) ont été partenaires pour développer le projet. Les animatrices appartenant à ces structures ont, suite à la formation, animé des groupes de parole dans leurs lieux d'intervention respectifs. Professionnelles de santé aussi bien qu'actrices de prévention dans des associations communautaires ou militantes dans des associations de quartier, leur point commun était d'être en lien avec des femmes en difficulté sociale, dans leur grande majorité. Depuis 2002, un effort particulier a été fait pour développer les actions dans les départements français d'Amérique, compte tenu de la prévalence importante de l'épidémie et de sa féminisation, particulièrement en Guyane.

Lors de la formation, un temps important est consacré à l'écoute, au savoir être, mais aussi, à travers des mises en situation, à une réflexion sur les représentations de la sexualité : que faire quand le partenaire refuse le préservatif ou n'autorise pas sa femme à prendre une contraception? Est-ce que l'homme a davantage de besoins sexuels que la femme et est-ce que la pénétration vaginale est le seul moyen d'avoir du plaisir pour les femmes?

Un autre objectif est de former les stagiaires à animer des groupes de parole qui se déroulent dans le temps (sept semaines) et sont basés sur le partage d'expérience. Ces groupes de parole sont l'occasion d'aborder la connaissance du corps et de son fonctionnement, de prendre conscience de tous les risques sexuels, de mieux connaître tous les moyens de protection. Ce sont les femmes elles-mêmes qui déterminent, de façon spontanée, les thèmes de discussion en fonction de leurs préoccupations. L'objectif des animatrices est que, à l'issue des groupes de parole, ces femmes soient capables de développer des initiatives personnelles ou collectives autour d'elles. Ainsi, certaines ont organisé des réunions dans leur quartier pour partager avec d'autres 
ce qu'elles avaient appris, elles ont expérimenté le théâtre forum ou monté des expositions sur la santé sexuelle et les droits des femmes. Plus modestement, nombreuses sont celles qui ont organisé, à la mode tupperware, des petites rencontres à leur domicile pour échanger avec des amies ou des voisines, afin de ne pas garder pour elle des informations qu'elles jugeaient importantes pour le bien-être des femmes.

\section{Les constats}

\section{La place des femmes, le rapport à la sexualité et à la prévention}

\section{Le programme Réduction des risques sexuels}

Dans le cadre de ce programme, les groupes sont très souvent constitués dans des structures, les femmes se connaissent avant parce qu'elles vivent dans le même lieu, participent à la même activité sociale d'alphabétisation ou de réinsertion. Mais le fonctionnement de ces groupes crée des liens différents permettant rapidement une parole libre, ce qui les surprend elles-mêmes : " jamais je n'aurais pensé pouvoir parler de tout ça» (sexualité, plaisir, corps, mais aussi violence dans le couple, manque d'autonomie des femmes...). La dynamique du groupe s'appuie sur les échanges d'expériences de vie et d'histoires singulières dans lesquelles les femmes retrouvent un peu de leur propre vie. À travers les 800 groupes réalisés et les 8000 femmes rencontrées, nous avons pu noter le poids des violences comme frein à l'appropriation de la prévention et plus largement à l'autonomisation : pas un groupe dans lequel cette question n'ait été soulevée. Dans le document décrivant l'évaluation du programme réalisé en Îlede-France, il est noté : « La formation apparaît comme pertinente pour atteindre les objectifs fixés, notamment en terme d'amélioration des connaissances et d'augmentation des aptitudes à aborder les thématiques du corps, de la sexualité, des risques sexuels... Elle correspond également aux besoins identifiés par les participantes en terme de réassurance par rapport aux questions liées à la sexualité " [17]. Dans cette même évaluation, les animatrices qui ont réalisé des groupes de parole soulignent leur intérêt pour cette forme de travail.

Le travail dans les groupes intègre une dimension revendicative autour du droit au respect et à l'égalité. Pour de nombreuses femmes le droit à disposer de son corps n'est pas une dimension facile à imposer au quotidien. Pour que les groupes se déroulent dans l'esprit du programme, il faut transmettre ses objectifs aux animatrices, les savoirs être sur lesquels il s'appuie. Les animatrices apprennent à travailler à partir des savoirs des personnes et de leurs représentations, et ont pour objectif, dans un cadre d'écoute et de non-jugement, de transmettre des éléments de connaissance, de donner la possibilité aux femmes de réfléchir aux situations de risque auxquelles elles sont confrontées, et ainsi favoriser une prise de conscience et peut-être un changement de comportement. Ce double niveau d'intervention exige tout d'abord de discuter avec les animatrices de la gêne qu'elles peuvent ressentir en abordant les questions autour de la sexualité. II faut également travailler le rapport à l'autre dans la fonction d'" acteur de prévention ". On leur demande aussi de reconnaître aux femmes participant aux groupes le droit de prendre des risques, d'accepter des choix de protection qui ne sont pas les recommandations reconnues par la santé publique. Ce qui est acceptable pour soi, nous ne l'acceptons pas toujours, en tant qu'acteur de prévention pour les personnes dont on pense avoir « la responsabilité ». Cette double exigence, a été source de difficultés : peur d'être responsable de la prise de risque des personnes, de ne pas être comprise, de ne pas jouer le rôle attendu de référence aux bonnes pratiques.

Le parti pris du programme est effectivement de positionner l'acteur de prévention dans le respect de l'autonomie des personnes et de leur cheminement. La décision que prendra la personne, après avoir pu bénéficier des informations et des espaces d'échanges, est la meilleure et probablement la seule qu'elle puisse prendre à ce moment-là. Ce qui ne préjuge pas de ses possibilités à modifier cette décision à l'avenir en fonction de facteurs qui peuvent être tout à fait indépendants de notre action. Par exemple, certaines femmes, parce que leur situation économique s'est améliorée, se sont senties dans une situation plus favorable pour imposer des exigences à leur partenaire. L'expérience acquise par les animatrices dans le cadre de ce programme a eu un impact sur leur manière d'intervenir de façon plus générale. L'évolution du positionnement de l'animatrice au regard de son rôle en tant qu'actrice de prévention a pu être à certains moments source de tensions avec d'autres membres de l'équipe. La mise en œuvre du programme a eu un impact institutionnel certainement sous-estimé. Une des leçons à tirer est qu'elle doit être portée par l'équipe entière : les modifications de positionnement et de méthode d'intervention qui sont provoquées par la formation et la mise en œuvre du programme doivent être anticipées pour ne pas créer un conflit entre l'animatrice et les autres membres de la structure.

\section{Le programme Frisse}

Dans ce programme, l'accent est mis sur l'intériorisation des règles et des normes qui encadrent la sexualité humaine, la question étant d'intégrer à ces codes de conduites des modes de prévention adaptés aux marges de manœuvre réelles des femmes. Le temps de la formation est l'occasion de travailler sur les rôles des hommes et des femmes, les représentations de l'amour, du couple et de la fidélité en tant qu'obstacles à la réduction de l'ensemble des risques liés à la sexualité. L'idée est que l'intériorisation des normes n'équivaut pas à son acceptation et qu'il est possible d'agir sans pour autant se mettre en danger [18]. 
De 1999 à fin 2006, 410 femmes ont participé à des sessions de formation-action. Elles ont de 18 à 65 ans, résident majoritairement en France métropolitaine et sont originaires de 24 pays différents. Parmi elles, 201, inscrites à titre professionnel, sont infirmières, sages-femmes, médecins généralistes, assistantes ou conseillères sociales, éducatrices spécialisées, animatrices de prévention ; 209, inscrites à titre personnel sont étudiantes, enseignantes, mères au foyer. Elles viennent chercher des informations pour elles-mêmes et se sentent « légitimes pour transmettre des messages" à leurs proches. Les professionnelles apprécient "d'avoir le temps de penser, de réfléchir sur soi, nos conceptions, nos pratiques", de découvrir "un autre mode de relation valorisant l'autre dans sa recherche d'outils sans imposer des modèles". Pour certaines, il reste cependant "difficile d'accepter que rien n'est sûr à $100 \%$ ", "difficile de comprendre où en est l'autre et d'accepter que le risque fait partie de la vie".

Toutes apprécient la non-mixité qui permet de « parler librement, d'avoir une réflexion sur la sexualité des femmes, d'exister, de se reconnaître une valeur", "d'apprendre à expliquer simplement comment est fait le sexe d'une femme", "d'avoir une information claire sur les moyens de contraception et de protection des maladies sexuellement transmissibles et le VIH, en laissant le choix à la personne en fonction de son contexte de vie ». La diversité des cultures d'origine permet des échanges au-delà des représentations parfois caricaturales des habitudes de " l'autre " : techniques d'assèchement ou de lubrification vaginale censées être pratiquées "chez nous en Afrique", alors que chacune de ces "coutumes" est identifiée comme une norme par des femmes originaires du même pays. Ce type d'apports illustre la diversité des codes de conduite et les risques qui en découlent, et permet de faire le parallèle avec les risques d'ulcération des muqueuses vaginales liés à l'usage abusif de gels intimes et/ou quotidien de tampons hygiéniques en Europe.

La diversité des pratiques évoquées spontanément par certaines "fait découvrir des mondes inconnus ", "facilite la levée des tabous" et permet de comprendre "qu'il faut partir des pratiques pour identifier et relativiser les risques». Ces échanges soulignent aussi des manques, notamment en matière de suivi gynécologique et de prévention des IST chez les lesbiennes et les transexuel-le-s, initiant un travail réflexif sur une réduction des risques adaptée à leurs pratiques. Conséquence directe de constats effectués au fil des sessions, la parution de "Gouixx "6 (brochure de réduction des risques en direction des gouines et des femmes qui font du sexe avec des femmes) est un exemple de la démarche de Frisse : accompagner un projet jusqu'à l'autonomie, puisque les groupes de discussion autour de cette brochure se déroulent en dehors du programme. Un travail analogue avec et en direction des transexuel-le-s est en cours.

\footnotetext{
Titre de la brochure publiée en 1000 exemplaires, financés par Frisse sur la ligne * Communication * de la DRASS Rhône-Alpes en janvier 2006
}

\section{Et les hommes?}

Notre intervention questionne en permanence la relation entre les hommes et les femmes : la question des places sociales respectives, mais également la façon de vivre l'amour, la sexualité, les relations au pouvoir. L'enjeu d'un travail sur la masculinité et la féminité avec les femmes, mais aussi avec les hommes, s'est posé dès le départ. Les deux programmes ont chacun leur façon d'intégrer cette question.

Pour le programme Frisse, agir avec et auprès des femmes n'a jamais été conçu comme une exclusion des hommes. C'est impossible dans un monde où la norme est l'hétérosexualité et où, pour faire la preuve de leur virilité, les hommes sont censés multiplier les " conquêtes " pour libérer leurs pulsions sexuelles, être sûrs d'eux et tout savoir sur la sexualité, alors que «les femmes bien " sont censées se laisser séduire et initier par les hommes. Et que la promotion du « tout capote " place hommes et femmes entre désir et droit au plaisir, maîtrise de la reproduction et prévention des maladies sexuellement transmissibles. Ainsi, les pressions sociales à la virilité et à la féminité sont évoquées de manière réflexive en faisant le lien entre sexisme et homophobie, notamment autour de livres pour enfants qui bousculent les modèles de construction sociale des filles et des garçons. Par ailleurs, bien qu'il n'existe pas en France de discours public valorisant les pratiques de non-pénétration chez les hétérosexuels, au contraire du discours de prévention en direction des homosexuels ${ }^{7}$, celles-ci sont abordées en termes de plaisir et de réduction des risques, ne serait-ce que pour différer la pénétration, les problèmes d'érection et leurs effets sur la relation. Le partenariat de Frisse avec Couples contre le sida ${ }^{8}$ donne ainsi naissance à un support de paroles sur les " techniques " possibles en l'absence de préservatif ou d'érection : " la boîte à malices ". II s'agit d'une petite urne en carton dans laquelle sont déposées les réponses à cette double question. Elles sont ensuite dépouillées et commentées à l'occasion de petits groupes de paroles. De plus, parallèlement aux formations-action, Frisse mène des actions ouvertes à toutes et à tous : conférences-débats, stands inter-associatifs, ateliers, journées thématiques ${ }^{9}$ et formations d'équipes mixtes. En outre, l'existence ponctuelle du groupe GHRISSE ${ }^{10}$, peut illustrer la difficile implication des hommes sur le terrain mouvant des relations, des émotions et des représentations de la virilité, puisque ce groupe est composé d'hommes qui se perçoivent en décalage avec les modèles de la masculinité et ne sont "pas prêts à se mobiliser auprès d'hommes trop différents ". L'idée est alors de les considérer comme des "déviants positifs" [19] et de les impliquer dans un travail de réflexion et d'expériences

\footnotetext{
Fernandez C. $3^{e}$ Journée régionale Ancic-Mouvement français pour le planning familial, septembre 1998. Ce médecin est l'une des personnes ressources de Frisse.

Association nationale auprès des publics multipartenaires dont le siège social est a Lyon, impliquée dans le pôle de personnes ressources du programme Frisse.

9 Exemple de thématiques : femmes, produits et sexualité ; prévention jeunes, sexisme et difficultés de prévention ; anthropologie des migrations ; représentations du masculin et du féminin ; alcool et prises de risques.

10 Garçons, hommes, réduction des risques et sexualité.
} 
valorisant des modèles alternatifs aux stéréotypes de la virilité. La formation et l'accompagnement en 2007 d'un groupe mixte de 16-20 ans, les "frissons", qui conçoivent et réalisent des actions auprès de leurs pairs, se situent dans cette perspective.

Pour le programme Réduction des risques sexuels, le choix d'espaces de paroles réservés aux femmes a été motivé par deux raisons : d'une part laisser émerger leur parole concernant les facteurs d'exposition aux risques tels que la violence conjugale, le manque d'autonomie financière, les représentations sur la place de la femme et de l'homme dans la sexualité. D'autre part, nous avons expérimenté depuis de nombreuses années, dans le cadre des animations autour de la sexualité en milieu scolaire, que la mixité dans les groupes était un frein à la prise de parole pour de nombreuses filles qui ne se sentaient pas autorisées à s'exprimer en présence des garçons, ou, à l'inverse, entraînait des attitudes de provocation. Même si l'on peut penser qu'une part de ces comportements est liée à la période de l'adolescence, nous avons cependant fait l'hypothèse que les femmes parleraient plus facilement entre elles, hypothèse qui s'est vérifiée car nombreuses sont celles qui ont souligné qu'échanger entre femmes est nécessaire. Mais elles nous ont rapidement fait remarquer que nombre de freins à l'adoption d'une prévention plus efficace venait des hommes, que beaucoup de femmes étaient contaminées par leur mari, sans qu'elles puissent intervenir sur le comportement de celui-ci et que, donc, il faudrait faire la même chose avec les hommes.

C'est pourquoi, nous avons décidé de diversifier notre intervention et développé un programme mixte. Les formations à l'animation de ce nouveau programme s'adressent à des hommes et à des femmes issus d'associations de prévention et de lutte contre le sida, d'associations communautaires ou d'insertion sociale. Ils animent des groupes auprès de leur public, et tout particulièrement auprès d'hommes dans les dispositifs d'insertion sociale ou auprès de jeunes dans les lieux de " réinsertion scolaire ». Les hommes et les femmes travaillent dans un temps non mixte puis mixte, ce qui permet de garder ce temps d'expression " entre soi ", avec l'avantage de permettre immédiatement après de confronter les représentations respectives des hommes et des femmes. La nature du travail a changé : le cœur de l'intervention devient la question de la communication entre les hommes et les femmes, les échanges portent sur les rôles respectifs, et sur ce qui fait obstacle à cette communication et aux relations de respect mutuel. La dimension de la négociation est beaucoup plus présente que dans les groupes de parole de femmes et nous avons pu constater une double évolution. D'une part les femmes mesurent que les hommes se posent beaucoup de questions, qu'ils ne sont pas aussi sûrs d'eux qu'ils veulent bien le laisser paraître et que, d'autre part, la communication est possible dans le cadre des groupes. Même si cela ne remet pas en cause la réalité des conditions de vie au quotidien, cette expérience semble renforcer leurs capacités à affronter ces réalités.
Pour les hommes, la découverte du vécu collectif des femmes, hors du contexte des relations interpersonnelles qu'ils peuvent vivre, leur permet de prendre conscience de la nécessité d'une plus grande prise en considération du point de vue des femmes et d'un renforcement des relations hommes/femmes. La possibilité d'avoir un espace de parole collectif " entre hommes" pour aborder les difficultés rencontrées dans leurs relations avec leurs partenaires apparaît aussi dans les retours des groupes comme une expérience importante.

\section{Conclusion}

Notre travail de terrain semble montrer que le renforcement des capacités des femmes à faire face aux risques relève à la fois de l'acquisition de connaissances, de la prise en compte de leurs désirs et de leurs droits, mais aussi des moyens qui leur sont offerts : les espaces de parole mis en place par chaque programme répondent, selon des modalités différentes, à des besoins de verbalisation et de partage autour des enjeux liés à la sexualité. Pour les femmes, il semble que " l'entre soi » facilite la mise en commun d'un vécu qui " sort difficilement du privé " et du paradigme du couple, et favorise le " prendre soin de soi ». Et le paradigme du couple renvoie à la nécessaire implication des hommes!

Cette démarche est loin d'être généralisée en France, malgré la volonté qu'à eue le ministère de la Santé, en 1999, de mettre en place un programme " en direction des hommes incluant homosexuels et hétérosexuels " [9]. Or, les hommes ont peu l'occasion d'exprimer leurs difficultés, d'autant que cette expression peut être interprétée comme une faiblesse puisqu'ils sont censés tout savoir sur la sexualité.

Cette perspective est toujours d'actualité, même si elle doit être revisitée à la lumière des expériences de mixité des programmes de Frisse et du Mouvement français pour le planning familial.

Comme cela a été proposé aux femmes, il faut développer dès aujourd'hui des programmes d'implication des hommes en partant de leurs sentiments et de leurs opinions ${ }^{11}$.

La proposition serait de créer des espaces de paroles et de mener des actions expérimentales qui pourraient s'inspirer du bilan des " cliniques masculines " de PROFAMILIA en Colombie, qui offrent des espaces de discussion et des options contraceptives masculines telles que le préservatif et... la vasectomie ${ }^{12}$. Dans une vision plus globale de la santé masculine, ces «maisons des hommes " pourraient rendre plus abordables des questions telles

\footnotetext{
11 Dans ce sens, le Centre international Margaret Sanger et la Fédération internationale pour la planification familiale ont mis au point un exercice qui peut favoriser l'expression des opinions et préjugés des hommes sur les femmes, en les confrontant á un certain nombre de déclarations auxquelles ils doivent réagir : * un homme célibataire a-t-il le droit d'avoir plusieurs partenaires? * * Le recours d'une femme à une méthode de contraception à l'insu de son mari constitue-t-il une trahison? n. USAID Comité sur les hommes et la santé de la reproduction. http://www.fhi.org/fr/RH/Pubs/Network/v18_3/NW183ch1.htm 12 Cf. (USAID) ibid
} 
que les dysfonctionnements sexuels, le cancer de la prostate, le cancer des testicules...

Dans la même dynamique, il faut développer des campagnes qui mettent en scène des attitudes positives. La déconstruction de l'assignation à un rôle prédéterminé demande de pouvoir s'identifier à d'autres modèles. II faut donc que les acteurs de prévention, les politiques de santé publique travaillent dans ce sens.

La conférence sur le sida qui s'est déroulée à Toronto en août 2006 a été l'occasion de présentations concernant de nouveaux outils de prévention, notamment le diaphragme et les microbicides. Les résultats de ces études, quels qu'ils soient, ne doivent pas nous faire oublier que les marges de manœurre des femmes sont d'autant plus étroites que les mentalités évoluent très lentement. Dans cette perspective, le recours au diaphragme reste une méthode barrière appréciable en terme individuel de réduction des risques pour celles qui l'adoptent lorsqu'il leur est impossible d'imposer à leur partenaire un préservatif masculin ou féminin ${ }^{13}$. Cette mobilisation des ressources et des énergies qui peut donner aux femmes plus d'autonomie ne doit pas se faire au prix de l'abandon des programmes incluant une approche " genrée ". Elle devrait au contraire s'accompagner d'actions de responsabilisation avec et auprès des hommes.

Si les femmes ont besoin de nouveaux outils pour renforcer leurs capacités à se protéger, elles ne pourront y avoir recours et s'autoriser à les utiliser que si, en parallèle, sont abordées les questions des droits, de la lutte contre les violences et sont mises en place des mesures volontaristes pour renforcer l'égalité et le partage des responsabilités entre hommes et femmes.

Ces exigences demandent un engagement fort, à la fois politique et financier, des états et des organismes internationaux, avec des moyens à la fois pour accompagner dans la durée des interventions de terrain mais également pour développer des recherches en sciences sociales.

\section{Références bibliographiques}

1. Prévention du Sida. Questions de Femmes. Paris : Agence française de lutte contre le sida et Centre national d'information et de documentation des femmes et des familles, décembre 1990.

2. $B E H 1997, n^{\circ} 34$.
3. Besson C. Cohorte de femmes infectées par le VIH, recrutées de fin 93 à fin 97 dans 10 pays d'Europe. In Actes du colloque Femmes et infection à VIH en Europe. Paris : CFES, 1999.

4. Bochow M. Les déterminants des comportements à risques. Revue des sciences humaines et sociales $1993 ; 39: 47-55$.

5. Giraud M et coll. Analyse des comportements sexuels aux Antilles et en Guyane, Acsag, 1994.

6. Hamblin J, Reid E. In Les femmes, l'épidémie d'infection par le VIH et les droits de la personne humaine : un impératif tragique. New York: UNDP publications issues, 1991.

7. Reducing Women's Vulnerability, Unaids, Washington, 1995.

8. Baier EG. De l'impact du VIH/sida sur les familles/communautés rurales et de la nécessité de concevoir des stratégies multisectorielles en vue de prévenir la pandémie et d'en atténuer les effets dans les zones rurales (orientation Afrique). Rome : FA0 Publications, janvier 1997.

9. Arnaudiès $M$. Une stratégie de réduction des risques sexuels en direction des femmes. Enjeux mars $1999 ; 93: 34$.

10. Plaza M. Gynécologues, femmes et sida. Du discours médical sur la femme aux pratiques préventives avec les femmes. In Sexualité et sida. Paris : Anrs, collection Recherches en sciences sociales, 1995, p. 311.

11. Spencer B. Où sont passées les relations hommes-femmes ? Journal des Anthropologues, Femmes et sida 1997, p. 73.

12. Spencer B. Contexte normatif du comportement sexuel et choix des stratégies de prévention. Population sept-oct $1993 ; 5: 1411-1436$.

13. Apostolidis T. Les représentations sociales de la sexualité et les comportements face au sida dans une population de jeunes adultes, une comparaison France/ Grèce. In Sexualité et sida. Paris: Anrs, collection Recherches en sciences sociales, 1995, pp. 247-250.

14. Rao Gupta Geeta. Genre, sexualité et VIH. Journal du sida et de la démocratie sanitaire, numéro spécial Anrs information, automne, p. 11-18.

15. Preciado B. Le manifeste contra-sexuel. Paris : Editions Balland, 2000.

16. Mossuz-Lavau J. Rapport d'évaluation du programme de réduction des risques sexuels en direction des femmes. Paris: Centre d'étude de la vie politique française, 201.

17. Laurent-Beq A, Fabre C. Évaluation de la formation d'animatrices de prévention dans le cadre du programme de réduction des risques sexuels en direction des femmes. Session du Val d'Oise 2004-2005, Bureau d'étude GRES médiation santé.

18. Nicole-Claude M. Quand céder n'est pas consentir. In L'arraisonnement des femmes: essais en anthropologie des sexes, M Nicole-Claude, Ed. Paris : EHESS, collection Cahiers de l'Homme, 1985, 169-243.

19. Barker G. Dimensions de la vulnérabilité des jeunes face à l'épidémie. Séminaire Vulnérabilité et prévention des MST $/ \mathrm{NI} /$ sida : des paradigmes aux nouvelles perspectives. Rio de Janeiro, 8-10 décembre 2004.

\footnotetext{
13 - Cette assertion n'engage que la responsabilité des auteures de cette contribution. En effet, les résultats de l'essai randomisé MIRA (test du diaphragme associé à un gel - Replens - pour la prévention de la transmission du VIH) ne montrent aucun effet bénéfique : c'est-àdire, il n'y a aucune différence significative entre le bras utilisant le diaphragme et auquel il est conseillé d'utiliser aussi des préservatifs et le bras qui reçoit le conseil d'utilisation des préservatifs seulement. Voir Padian NS et al. Diaphragm and lubricant gel for prevention of HIV acquisition in southern African women: a randomised controlled trial, The Lancet, 2007 ; 370 : 251-261 (Note des coordonatrices). *
} 